\title{
Design of Measuring System of ASC (Acupuncture Spot Current) for Constitutional Diagnosis
}

\author{
Un-ho, $\mathrm{Ji}^{1, \text { a }}$, Soon-yong, Chun ${ }^{2, \mathrm{~b}}$ \\ ${ }^{1}$ Dong-Yang University, Korea, ${ }^{2}$ Dong-Yang University, Korea \\ ajiunho@hotmail.com, ${ }^{b}$ control@dyu.ac.kr
}

\begin{abstract}
Keywords: ASC(Acupuncture Spot Current), sasang medicine, meridian point/acupuncture spot, constitution diagnosis.
\end{abstract}

\begin{abstract}
In Sasang Medicine, which is a part of Eastern Traditional Medicine, human body is classified by constitutions for diagnosis and treatment. In this study, a fundamental study on the ways and means of measuring the constitutions is classified in Sasang Medicine. A method of measuring ASC (Acupuncture Spot Current) through acupuncture spots on human body is proposed, together with a way to extract characteristic points of measured signals. Based on the characteristic analysis of the measured ASC, it is able to define the measured signals of experimental targets through classification, and the defined characteristics of ASC were determined to be able to be utilized in developing systems for medical constitutional classification.
\end{abstract}

\section{Introduction}

Just as a patient's respiratory status, blood pulse, blood pressure, body temperature etc are measured for diagnosis in modern medicine, in Eastern Traditional Medicine, a patient's physical state is diagnosed through the diagnostic actions of looking, asking, listening and touching[1][2]. Lots of symptom information is collected using every Eastern Traditional Medical method to procure objectivity in diagnosis and for more accurate diagnosis. The analysis is based on the collected symptoms and the judgment of a disease of made.

Likewise, when treating a patient, Eastern Traditional Medicine makes a difference between prescriptions according to a patient's constitution and this is based on sasang medicine. In sasang medicine, the treatments are distinguished according to the differences clarified by the constitutions. At this point, the criterions used to distinguish constitutions are 'sungjung' which means one's usual nature or dispositions and 'yongmosaki' which is based on one's body frame, appearance and behavior[3]. However, a patient's 'sungjung' and 'yongmosaki' are hard to be figured out as both needs to be analyzed at close quarters for a long time. To determine the constitutions, Eastern traditional medical clinics enhance the accuracy of constitutional diagnosis by applying various methods simultaneously.

In the methods of constitutional diagnosis, a variety of distinguishing methods are multilaterally analyzed to clarify diagnosis such are methods using an evaluation form of constitution distinguishing questions, a patient's appearances, blood pulse diagnosis, acupunctural reaction tests, drug reaction tests, constitution analysis instruments and a patient's disposition revealed during diagnosis. However, it requires a considerable amount of time and diagnostic procedures to apply all the multilateral methods listed above and thus the practical application isn't easy and using a few simple set of diagnosis leaves us results with a very low accuracy for making determinations.

Hereupon in this study, I carried out a basic study to easily, quickly and accurately diagnose constitutions in Easter Traditional Medicine. For the method to diagnose constitutions, I propose a method for measuring bioelectric conduction characteristics and a characteristic classification method through experiment.

\section{Devices and results}

Studying characteristics through human bodies' bioelectric phenomenon and electrical stimulus were actively implemented since the 1950s. Since Hodgkin's study of action potential, studies on bioelectric characteristics of skin[4-9], studies on mathematical models of skin and electrodes[10,11], 
and studies on stimulus parameters[12] etc were carried out. Such bioelectrical characteristics are diversely shown according to physical activities and individuals, and have been the foundation of bioelectrical signal measurement including EEG and ECG measuring for diagnosis in modern medicine. However, cases researched as medical means are mostly being based on modern medicine, and researches for Eastern medicinal diagnostic means are in poor status.

Basic studies as to body characteristics measuring methods which can be utilized for Eastern medical diagnoses and prescriptions were carried out. Measuring of bioelectrical conduction characteristics signals was implemented through 'acupunctural spots' used at Eastern medical diagnoses and treatments. The bioelectrical conduction characteristics of 'acupunctural spots' differ from those shown on other skin surfaces [13-15].

1. Bioelectrical Conduction Characteristics Measuring System

In this research, I designed a measurement system for bioelectrical conduction characteristics test, and Fig. 1 represents the structure of the designed system.

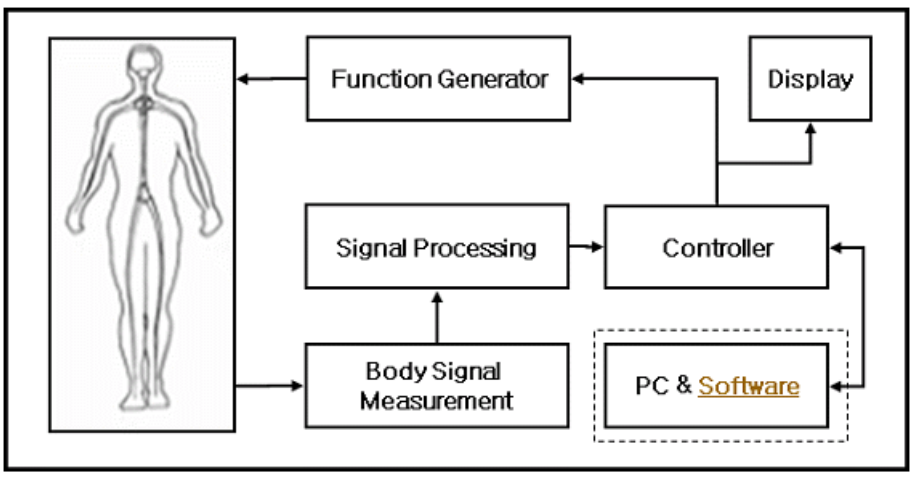

As you can see from Fig.1, the system is composed of the control part using a micro-processor, a measurement part to measure the body signal, the basis signal generation part for measurement, the display and the communication part.

Fig. 1. Block diagram of Measurement System

\subsection{Control Part}

Atmel's Atmega128 process was used for the control part. Atmega128 has ADC for analog signal measurement and control signal generation and an interrupt for peripheral devices control.

1.2 Body signal measurement part

The body signal set as a measurement element in this research is the bioelectrical conduction characteristics through 'acupunctural spots'. It measures the conduction characteristics of the electrical signals generated by a function generator, and the measured signal is composed of the conduction current change by body impedance change and the time data of which the basis measurement signal passes the body and turns on the transistor switch. The time data is derived from

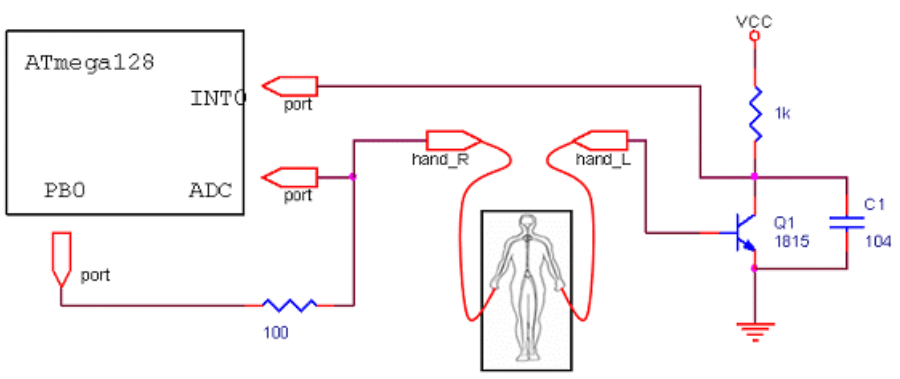
the response characteristics of the system due to the step signals generated by the control part. Such body information is measured through the ADC(Analog to Digital Converter), timer and interrupt functions included in the system. Fig. 2 is the structure diagram of the measurement part for body signal measurement.

Fig. 2. Block Diagram of Measurement Part 


\subsection{Body signal processing part}

In the signal processing part, signals of the required level are generated through amplification and filtering of the measured signal from the sensors. Fig. 3 represents the process and the Body signal processing part consists of a hardware method and a software method in this study. Fig. 4 shows the circuit of the amplifying part and the filtering part of the hardware of the body signal processing part[16].

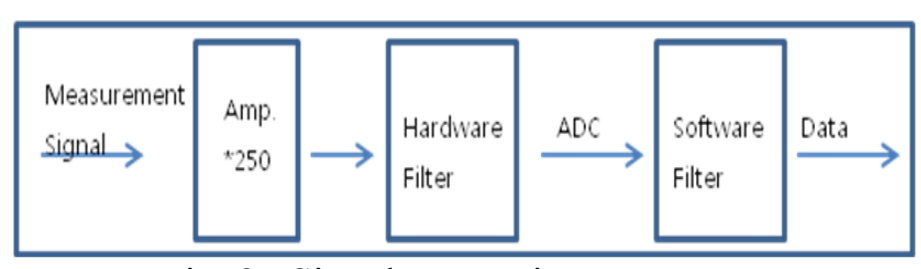

Fig. 3. Signal processing

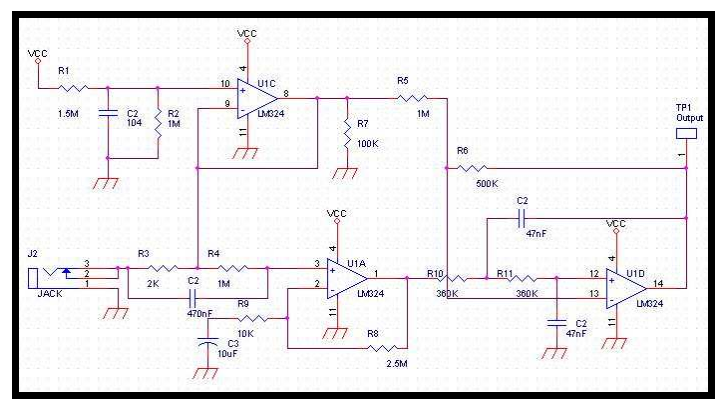

Fig. 4. Circuit for signal processing

\subsection{Function Generator}

The basis measurement signal input to human body for electrical conduction characteristics measurement is a Square Wave Type as shown in Fig. 5.

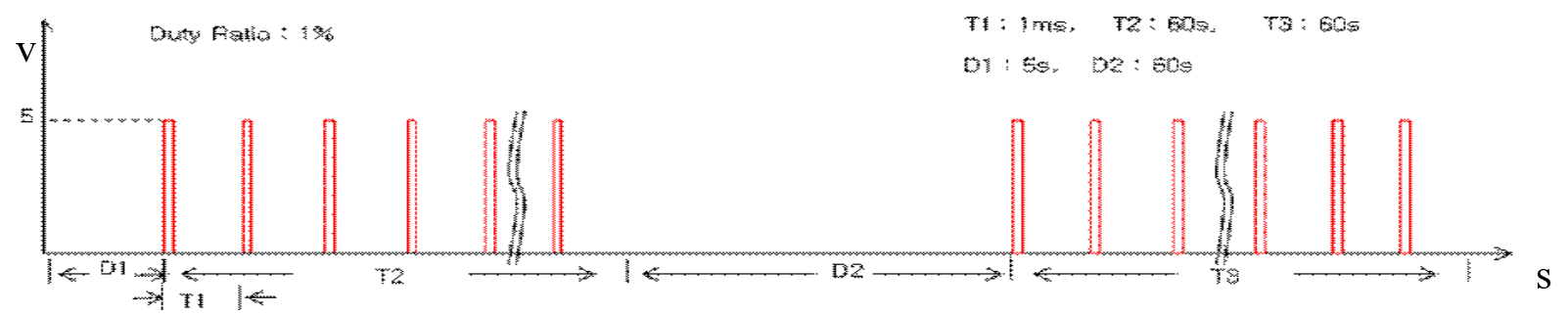

Fig. 5. Input Signal

\subsection{Display \& PC(personal computer) Software}

The display part basically displays the measured signals and information during the measurement, and the data transferred through serial port using RS-232 communication system is shown in Graphical Wave and stored in a storage media in the PC Software.

2. Bioelectrical conduction characteristics measurement experiment

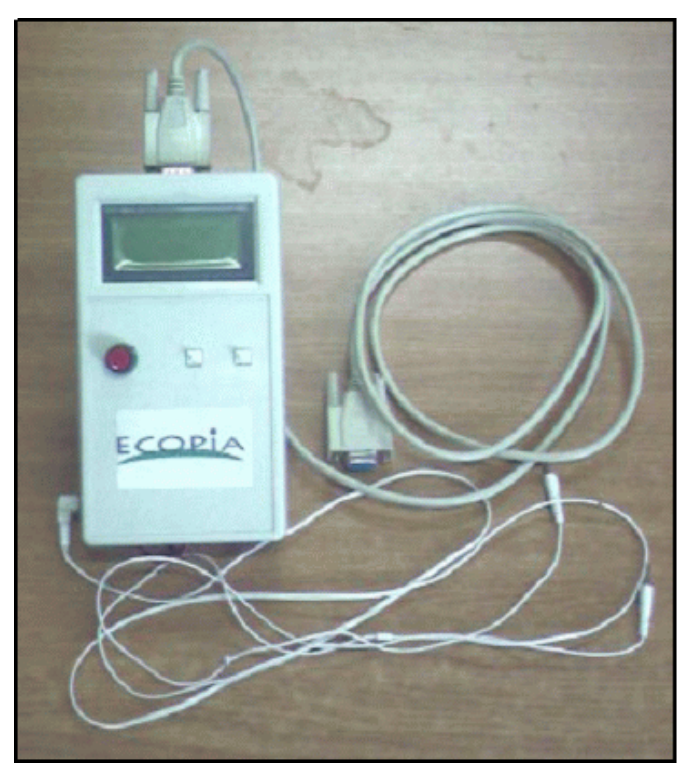

Measurement experiment was carried out using the bioelectrical conduction characteristics measurement system discussed in the first content of this paper. Fig. 6 shows the measurement system designed and produced.

The experiment was carried out in a laboratory made to block energy sources coming from external environment such as noise/electromagnetic waves capable of stimulating the subject during the experiment. It was based on a supposition that no external energy existed in the laboratory excluding minute electrical signals generated from the system. The electrical conduction characteristic measurement experiment was carried out on 'HapGok' acupuncture spot of a subject's right hand using his left palm as a basis.

Fig. 6. Measurement System 
The measurement was done in two perspectives as follows.

i) The amplitude of conduction current

ii) The response characteristic by the step input

\subsection{The amplitude of conduction current}

The conduction characteristics of which measurement experiment from 'HapGok' acupuncture spot was carried out using the designed measurement system are shown in Fig. 7.

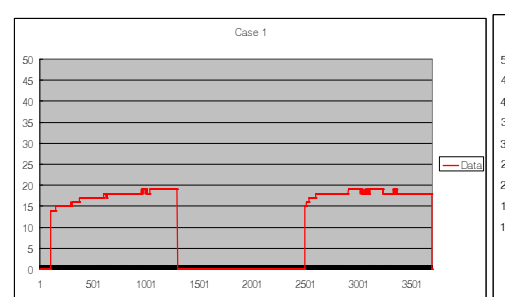

(a)

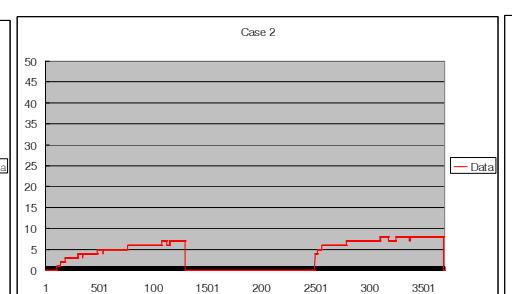

(b)

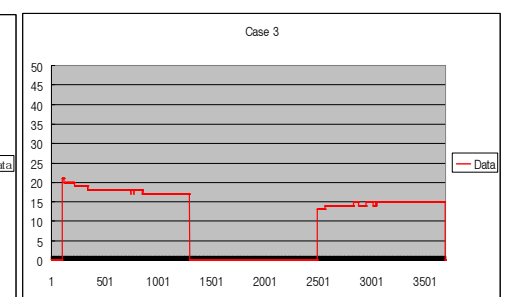

(c)

Fig. 7. Pattern of current (a) case 1, (b) case 2, (c) case 3

As represented in Fig. 7 the electric current conduction characteristics appear differently according to the physical status of each individual. The figure represents the conduction characteristics measured using the basis signal generated from the Function Generator discussed in 1.4 as they are the displays of the outputs of the Square Wave Type inputs.

The conduction characteristics were able to be identified in the measurement experiment result as the conduction current either increased in the first measurement basis signal section and maintained a constant amplitude in the second section (Case 1 and Case 2) or decreased in the first section and then remained a constant value in the second section(Case3).

\subsection{The response characteristic of step input}

Fig. 8 shows the system output characteristics of the step input signal.

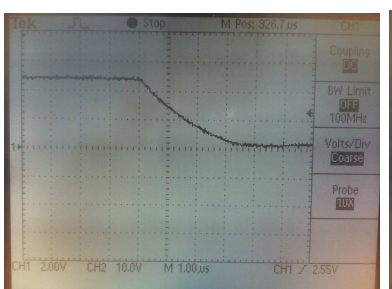

(a)

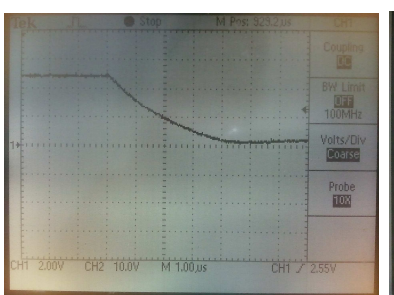

(b)

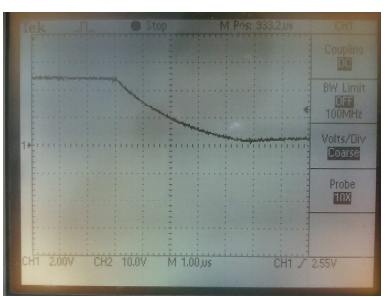

(c)

Fig. 8. Output by step input (a)case 1, (b) case 2, (c) case 3

The output characteristics are the characteristics of the signal measured in the collect end of a transistor in the resonse signal measurement system discussed in Fig. 2 and the output characteristics of step input differs according to the physical status.

The vertical axes represent the voltage level at the collect end and the horizontal axes represent time in the figure.

These characteristics are analysed as characteristics shown according to the change of RLC components which human body contains when modelling human body into an electric circuit, and from these characteristics the model of human body including measurement circuit is expressed as equation 1 below.

$$
a_{1} \frac{d y}{d t}+a_{2} y=v
$$

Here, a1, a2 can be defined as intrinsic parameters given according to a human's physical type. 
The expression of $y$ in equation 1 can be generalized as the equation 2 below.

$$
y=v e^{-\alpha t}
$$

From experimental output characteristics shown in fig. 8 , it can be expressed as $\mathrm{v}=5$ from equation 2, and $\alpha$ can be defined as a variable which can be obtained according to the physical characteristics of each human individual.

\section{Conclusion}

In this study I researched about measurement system for the classification and diagnosis of human constitutions. The designed system is a system to measure the electric conduction characteristics of a human body, and I measured the conduction characteristics using micro-currents passing through human bodies. Additionally, I measured the characteristics of transient response of step input using conduction current and transient response characteristic measurement circuit included in the system.

As a result of the measurement through the experiment, the response characteristic of human body including measurement system was able to be expressed in first order differential equation and 9 forms of conduction current characteristics, and the characteristics classification for classifying human constitutions from characteristics of inputs and outputs is determined to be possible. In the future when measuring data analysis research is carried on through continuous experimental research, human body system modeling for sasang constitution classification is determined to be possible.

\section{References}

[1] DeajeunMuneun. "Dosul Dongyang medicine", Nonjang: 1992

[2] Whan Kim, Oriental Medicine principle, SungBo: 1990

[3] ChanMin Kim, Sasang constitutional medicine, Academi: 2002

[4] T. Yamamoto and Y. Yamamoto, "Electrical properties of the epidermal stratum corneum", Med. Biol. Eng., Vol. 14, pp. 151-158, 1976

[5] T. Yamamoto, Y. Yamamoto and T. Ozawa, "Characteristics of skin admittance fot dry electrodes and the measurement of skin moisturization", Med. Biol. Eng. Comput., Vol. 24, pp. 71-77, 1986

[6] J. Rosell, J. Colominas, P. Riu, R. Pallas-Areny and J.G. Webster, "Skin impedance from 1Hz to 1MHz", IEEE Trans. biomed. Eng., Vol. 35, pp. 649-651, 1988

[7] D. Panescu, K.P. Cohen, J.G. Webster and R.A. Stratbucker, "The mosaic electrical characteristics of the skin", IEEE Trans. Biomed.. Eng., Vol. 40, pp. 434-439, 1993

[8] SoonYong Chun, UnHo Ji, H.Troy Nagle, "Observations of the current change on acupuncture spots at the meal time", IEEE CBMA204, 59, 2004

[9] SoonYong Chun, UnHo Ji, "Design for a Conductivity Measurement System”, Korea IEEK, 2009

[10] D. Panescu, J.G. Webster and R.A. Stratbucker, "A nonlinear electrical-thermal model of the skin", IEEE Trans. Biomed. Eng., Vol. 41, pp. 672-679, 1994

[11] S.J. Dorgan and R.B. Reilly, "A model for human skin impedance during surface functional neuromus-cular stimulation", IEEE Trans. Rehab. Eng., Vol. 7, pp. 341-348, 1999

[12] P.T. Kolen, "Transformer miniaturization for trans-cutaneous current/voltage pulse applications", IEEE Trans. Biomed. Eng., Vol. 46, pp. 606-608, 1999

[13] Korea institute of Oriental Medicine, "Research of meridian system I", Korea institute of Oriental Medicine: 1996

[14] Nakatani, Y. On the nature of the acupuncture points and meridians: J. Jap. Orient. Med. 3, 1953:39 49

[15] Haiso Yu-san. Meridians as enisaged from the study of skin conductance: J.Chin, Traditional Med. Feb., 1958:1 8

[16] John Enderle, "Biomedical Engineering”, Elsevier, 2005 